\title{
Cultura del Honor e Inteligencia emocional: ¿conceptos relacionados o incompatibles?
}

\section{Culture of Honour and Emotional Intelligence: Incompatible or related concepts?}

\author{
Esther López-Zafra ${ }^{1}$, Noelia Rodríguez-Espartal y $\mathrm{M}^{\mathrm{a}}$ Isabel Jiménez Morales \\ Departamento de Psicología, Universidad de Jaén, España.
}

(Recepción: Octubre 2008 - Aceptación: Noviembre 2008)

\section{Resumen}

En el presente estudio relacionamos dos conceptos, Inteligencia Emocional y Cultura del Honor; en ambos casos el aspecto emocional es muy relevante y creemos que pueden tener un papel en las relaciones de pareja.

Planteamos que ambos conceptos se relacionarían en sentido inverso, de forma que un mayor nivel de Inteligencia Emocional llevaría a otorgar una menor importancia a la Cultura del Honor y viceversa. Para estudiarlo se utilizó una muestra de 203 parejas heterosexuales que completaron un cuestionario. Entre nuestros resultados comprobamos que la dimensión Atención a las emociones está relacionada con la cultura del honor. Entre nuestras conclusiones planteamos que ambos conceptos están relacionados en alguna medida y que la congruencia en la valoración de la Cultura del Honor entre los dos miembros de la pareja también se relacionará con un nivel de Inteligencia Emocional mayor que en parejas donde no se produce esta congruencia.
\end{abstract}

Palabras clave: Cultura del Honor, Inteligencia emocional, congruencia en la pareja.

\begin{abstract}
In this study we relate two concepts, Emotional Intelligence and Culture of Honour; in both cases the emotional aspect is very important and we believe they may have a role in couple relations. We propose that both concepts would relate in reverse, so that an individual with a high level of Emotional Intelligence would give less importance to the Culture of Honor and vice versa. A sample of 203 heterosexual couples completed a questionnaire. Our results show that the dimension Attention to emotions is associated with the culture of honor. Among our findings we propose that the two concepts are related in some way and that congruency in the valuation of the Culture of Honor between the two partners will also deal with a level of Emotional Intelligence higher than in couples where there is not this congruence.

Keywords: Culture of honor, Emotional Intelligence, couple congruency.
\end{abstract}

1 Correspondencia a: Esther López-Zafra, Departamento de Psicología. Área de Psicología Social, Edif. D2, Campus Las lagunillas s/n, 23071, Jaén. Email: elopez@ujaen.es Teléfono: +34 953211990 Fax: +34 953211881 


\section{Introducción}

Los seres humanos como seres sociales, tenemos una gran necesidad de afiliación. Las relaciones cercanas y positivas con la pareja, la familia, los amigos y los compañeros de trabajo constituyen un aspecto fundamental en la vida de las personas porque influyen positivamente en su salud y su felicidad (Atchley y Britton, 2007; Bisquerra, 2000; Punset, 2006); pero existen también otro tipo de relaciones que pueden conllevar efectos negativos para la persona, como es el caso de las relaciones de pareja en las que dominan los celos o celotipia (esto es, se sobrepasa el deseo legítimo de amar a una persona y no compartirla con nadie, llegándose a los celos patológicos y obsesivos, Alcaide, 2002) y que pueden desembocar en violencia de género. En cualquier caso, las relaciones sociales y en este caso, de pareja, implican un componente emocional muy importante.

En este trabajo relacionamos dos conceptos que pueden jugar un papel importante en la violencia de género: la inteligencia emocional y la cultura del honor. Por un lado, la inteligencia emocional implica la capacidad de atender, comprender, y manejar las emociones propias y las de los demás; por otro, la cultura del honor se refiere a la percepción de que las acciones del otro miembro de la pareja influyen en el honor individual. Creemos que una persona que sea capaz de entender al otro, evaluará emocionalmente mejor las situaciones que se producen en la pareja y por ello, es posible que tenga en cuenta más aspectos en la relación, ofreciendo menor importancia a la cultura del honor. Pero veamos más detenidamente ambos conceptos para comprender la relación que pretendemos analizar.

\section{Cultura del honor y Relación de pareja}

¿Por qué ante una situación de ofensa o insulto emitimos reacciones emocionales diferentes? Y además, ¿por qué esta reacción emocional puede variar en función del tipo de ofensa?

El grupo de Cohen y colaboradores se planteó esta pregunta al comprobar que los habitantes del norte y del sur de Estados Unidos de América reaccionaban de forma bien distinta ante ofensas que podían referirse al honor, siendo mucho más violenta esta reacción en el sur de los Estados Unidos (Cohen, Nisbett, Bowdle, y Schwarz, 1996); además, el hecho de que este resultado se replique transculturalmente (Vandello y Cohen, 2003), nos lleva a distinguir y hablar de culturas de honor vs. culturas de no-honor.

En cuanto a la expresión de una mayor o menor cultura del honor, se han encontrado diferencias entre países, e incluso entre zonas del mismo país, observándose, en general, que los países colectivistas presentan una mayor cultura del honor (e.g. países del Mediterráneo, latinos y sudamericanos) frente a los individualistas (e.g. Estados Unidos, con diferencias entre el sur, con una mayor cultura del honor, y el norte) (López Zafra, 2007). La diferencia entre las culturas de honor con otras que podemos denominar culturas de no honor descansa en la saliencia y centralidad que estos temas del honor tienen sobre la interacción social cotidiana (Vandello y Cohen, 2003). Así, en los países en los que se producen influencias de esta cultura del honor se observan una serie de características respecto a la importancia que el honor tiene en cuanto a la relación de pareja. En concreto, los hombres pertenecientes a dichas culturas perciben que deben defender su honor aun usando la violencia porque, si permiten insultos y menosprecios estarán ofreciendo una imagen de debilidad y de incapacidad de proteger lo que es suyo, que no están permitidas dentro de estas culturas del honor (Shackelford, 2005). Además, se enfatiza el honor masculino otorgando una gran importancia a los roles de género tradicionales que animan y perpetúan la violencia contra las mujeres (López Zafra y Rodríguez Espartal, 2008), obteniendo mayores puntuaciones en masculinidad aquellas personas que otorgan una mayor importancia al honor que personas que puntúan el honor como menos importante, quienes a su vez puntúan menos en la identidad de género masculina (LópezZafra, 2008).

En resumen, el concepto de cultura del honor hace referencia al conjunto de normas culturales generadas por la historia, las leyes y la política social y que permiten la pena capital y el maltrato a 
mujeres y niños (Cohen, 1996), perpetuándose gracias a las interpretaciones que leyes y medios de comunicación hacen sobre actos relacionados con el honor (Cohen y Nisbett, 1997; López Zafra, 2007). La relación de este concepto con la violencia de género es evidente ya que, en muchos casos, este tipo de violencia se justifica por la percepción de que la pareja es propiedad del otro, lo que da lugar a situaciones de celos que provocan reacciones emocionales violentas ante la percepción de amenaza de abandono por parte de la persona amada.

Un aspecto de las relaciones de pareja muy relacionado con este concepto de cultura del honor es el de los celos. Diversos autores destacan su importancia afirmando que la primera causa de la violencia del hombre hacia la mujer han sido los celos o la amenaza de abandono de la esposa (Daly y Wilson, 1988; Buss, 2000); además, en muchos casos son vistos como un signo de amor (Barron, citado en Yela y cols., 2003). Según Buss (2000), los celos serían los mecanismos clave que se activan en respuesta a una amenaza real o percibida sobre una relación que la persona considera valiosa; además, estos mecanismos serían diferentes en hombres y mujeres, viéndose ellos más afectados por una infidelidad sexual, mientras que a ellas les afectan más las infidelidades con carga emocional. A pesar de estas afirmaciones, Gómez-Jacinto, Canto y García (2001) han encontrado que la infidelidad emocional afecta más, tanto a mujeres como a hombres, y que la respuesta de cada uno ante la situación provocadora de celos estaría modulada por las normas sociales y el rol de género. Además, se ha comprobado que la violencia relacionada con los celos se legitima más en culturas del honor que en culturas de no-honor (Grandon y Cohen, 2002; Vandello y Cohen, 2008; y Vandello, Cohen, Grandon y Franiuk, en prensa) y que, cuando esta violencia está justificada de algún modo por los celos, no se percibe de forma tan negativa, aceptándose en mayor medida que un hombre sea violento con su pareja en esta circunstancia (Puente y Cohen, 2003). En esta línea se encuentran también resultados que ponen de manifiesto la existencia de una relación directa y significativa entre celos y cultura del honor (López Zafra y Rodríguez Espartal, en prensa).

En este sentido, y considerando las diferencias en cuanto a cultura del honor en diferentes países y la relación de los celos con este concepto, Vandello y cols. (2008) compararon muestras de latinos y americanos del sur de Estados Unidos (cultura del honor) con norteamericanos y canadienses (culturas de no honor) y encontraron que las culturas del honor pueden permitir la violencia de género a través de las expectativas de la lealtad femenina ante esta violencia; que estas culturas del honor pueden apoyar, o al menos no condenar, la violencia contra la mujer legitimando su uso en defensa del honor masculino y que los celos juegan un fuerte papel atenuante en la valoración negativa de la violencia.

Por último, queremos destacar el hecho de que las parejas que tienen una opinión similar respecto a cuánto de importante es para ellos la cultura del honor, informan también de una relación más satisfactoria (López-Zafra y Rodríguez Espartal, en prensa), apoyando la idea de la congruencia en la relación de la pareja.

\section{Inteligencia Emocional}

Todo lo anteriormente comentado, nos hace pensar que las culturas del honor están muy vinculadas a un tipo específico de reacciones emocionales.

El desarrollo más reciente en el campo de las emociones lo constituye el constructo Inteligencia emocional (IE), que hace referencia a la interacción adecuada entre Emoción y Cognición, que permite al individuo un funcionamiento adaptado a su medio (Salovey y Grewal, 2005). Puesto que las personas difieren en el modo en el que son capaces de identificar, expresar, utilizar y regular sus sentimientos y los de otras personas (Mikolajczak, Luminet y Menil, 2006), en los últimos años se han realizado numerosas investigaciones sobre este concepto (IE) que han contribuido a arrojar un poco de luz sobre los beneficios que presenta ser emocionalmente inteligente en áreas muy diversas del funcionamiento humano (Extremera, Fernández-Berrocal y Salovey, 2006),

La evidencia existente hasta el momento indica que altos niveles de inteligencia emocional, se asocian a un mejor funcionamiento psicosocial (Salovey y Grewal, 2005), que incluye tanto factores 
intrapersonales (como por ejemplo, un mayor bienestar) como interpersonales (como por ejemplo, mejores relaciones interpersonales).

El estudio exploratorio realizado por Winters y cols., (2004), reveló que bajas puntuaciones en diferentes dimensiones relacionadas con la Inteligencia emocional (evaluada mediante el EQ-i), entre las que se encuentran, entre otras, la auto-conciencia emocional, la asertividad, el control de impulsos, o la solución de problemas, parecen asociarse con una alta tendencia al abuso y con comportamientos violentos hacia la pareja (Winters, Clift y Dutton, 2004). Por su parte, Amitay y Mongrain (2007) examinan los correlatos interpersonales de la IE en una muestra de personas con una historia de depresión, y observaron cómo los participantes que presentaban niveles superiores de Inteligencia emocional (evaluada mediante el MSCEIT), percibían a sus parejas como menos hostiles, críticos y recusantes. Inesperadamente, aunque las parejas de los participantes con alta IE informaron que ofrecían menos apoyo activo y directivo que las parejas de los participantes con baja IE, los participantes con alta IE percibían un mayor apoyo por parte de sus parejas que aquellos participantes con baja IE. Asimismo, las parejas de los participantes emocionalmente más inteligentes también informaron de una mayor responsabilidad y apertura a la experiencia, resultados que apoyan la hipótesis de que altos niveles de IE actúan como un factor protector en este sentido (Amitay y Mongrain, 2007).

Con respecto a la relación existente entre satisfacción e Inteligencia emocional los estudios realizados hasta el momento son escasos. En este sentido se encuentra el estudio realizado en 2001 por Schutte y cols., en el que los participantes debían estimar tanto su propia Inteligencia emocional como la de sus parejas. Los resultados mostraron que aquellos participantes que autoinformaban una mayor Inteligencia emocional, también autoinfomaban niveles de satisfacción significativamente superiores a aquellos participantes con baja Inteligencia emocional autoinformada. Además, los participantes que puntuaron a sus parejas como altas en IE, también informaron niveles de satisfacción superiores al resto.

Recientemente Smith, Heaven y Ciarrochi (2008), llevaron a cabo un estudio con el objeto de analizar la relación existente entre IE rasgo, patrones disfuncionales de comunicación y satisfacción en parejas heterosexuales. Los resultados obtenidos por los autores mostraron que las parejas más satisfechas con su relación, fueron aquellas que no evitaban las discusiones (es decir que abordaban sus problemas de pareja) y que informaban altos niveles de IE en sus parejas.

Con respecto a los efectos de la satisfacción con la relación sobre la percepción de Inteligencia emocional, Smith y cols. (2008) examinaron, tras dividir a las parejas en dos grupos (alta y baja satisfacción), que las parejas más satisfechas obtenían puntuaciones similares en sus autoinformes de Inteligencia emocional. En el caso de los hombres, se encontraron diferencias significativas en el nivel de correlación existente entre el nivel de IE autoinfomada por el marido y la estimación realizada por su esposa tanto en las parejas de baja como en las de alta satisfacción. Este efecto sugiere que las parejas satisfechas era más probable que se percibieran a sí mismas como con un nivel similar de IE en comparación con las parejas insatisfechas.

Cuando se analiza el nivel de correlaciones entre las puntuaciones realizadas por los participantes y sus parejas, se observan diferencias significativas en la percepción de IE entre parejas satisfechas e insatisfechas. Así, los miembros de las parejas satisfechas tienden a presentar unos niveles de Inteligencia emocional autoinformada más similar que el mostrado por los miembros de parejas insatisfechas.

Por último, examinaron si aquellos individuos que informaban una baja satisfacción también tendían a puntuar a sus esposas como "bajas" en Inteligencia emocional. En este caso, los resultados obtenidos no fueron significativos en el caso de los hombres, pero sí en el caso de las mujeres. Las mujeres insatisfechas tendían a puntuar a sus parejas más bajo en IE que a sí mismas, mientras que las mujeres satisfechas consideraban la Inteligencia emocional de sus maridos, superior a la suya propia. Este estudio permite concluir que las parejas más satisfechas son aquellas que no evitan el conflicto (es decir que hablan de sus problemas), que tienden a percibir los niveles de Inteligencia 
emocional de ambos como muy similares y que tienden a idealizar la IE de la pareja en alguna medida (Smith y cols., 2008).

Como hemos observado en estos estudios, el que los dos miembros de la pareja se percibieran con niveles similares de Inteligencia emocional, era más probable en las parejas más satisfechas (Smith y cols., 2008), lo que refuerza la hipótesis de la congruencia.

Según todo lo anterior nos planteamos que Cultura del Honor e Inteligencia emocional deben ser conceptos relacionados en sentido inverso, por lo que personas con mayor inteligencia emocional le otorgarán menor importancia a la cultura del honor y por ende, al honor masculino, incidiendo este aspecto en una relación más satisfactoria en la pareja, en donde será menos probable que se produzca violencia en el seno de la misma. Por ello, pensamos que en esta nueva línea de investigación, que intenta analizar la relación existente entre la Inteligencia emocional y diferentes dimensiones que tienen que ver con las relaciones de pareja, como son la satisfacción, la violencia, etc., incluir la cultura del honor podría ofrecer resultados cuyas implicaciones clínicas podrían ser muy importantes.

\section{Estudio empírico}

Nos planteamos la necesidad de abordar empíricamente la relación entre estos dos conceptos. En concreto, consideramos las siguientes hipótesis de trabajo:

Hipótesis 1.- Se producirá relación entre Inteligencia Emocional y Cultura del honor.

En concreto, creemos que las personas con mayor inteligencia emocional otorgarán menor importancia a la cultura del honor que las personas con menor inteligencia emocional. Asimismo, personas que valoran mucho la idea del honor puntuarán menos en I.E.

Hipótesis 2.- Se producirán diferencias en inteligencia emocional en función de la valoración que los dos miembros de la pareja otorguen a la cultura del honor.

Creemos que cuando los dos miembros de la pareja tienen una percepción similar respecto a la cultura del honor, sobre todo si se le otorga menor importancia, su inteligencia emocional será mayor que en el caso de que ambos tengan una opinión distinta sobre la cultura del honor.

\section{Método}

Para este estudio se estableció como criterio que debían participar parejas de diferentes edades. El requisito imprescindible es que fueran parejas estables que llevaran un tiempo mínimo en la relación (6 meses) y que tuvieran intención de continuar con dicha relación en el futuro. No tenían que ser parejas casadas aunque sí heterosexuales.

\section{Participantes}

En este estudio participaron 406 personas (203 mujeres y 203 hombres que formaban 203 parejas) procedentes de distintas localidades de España. La edad media fue de 34.31 (S.D. = 14.46) con un rango de edades de 16 a 80 años.

\section{Instrumentos}

Para la realización del estudio empleamos un cuestionario al cual las personas respondían de manera individual y que contenía las siguientes escalas:

\section{Escala de la Cultura del Honor}

Esta escala ha sido elaborada por López-Zafra (2007), para medir Cultura del Honor como la actitud o predisposición a otorgar una importancia clara a la defensa del honor. Está compuesta por 17 ítems que se puntúan mediante una escala tipo Likert de 5 puntos. Conforman tres factores (Honor Individual, $\alpha=.71$; Sociedad y Leyes en torno al honor, $\alpha=.77$; y Legitimidad del uso de la violencia ante una ofensa, $\alpha=.83$ ). Además se puede obtener un índice global sobre la Cultura del 
Honor, con una fiabilidad en nuestro estudio de $\alpha=.82$.

Escala sobre Inteligencia Emocional

Esta variable se define como el metaconocimiento que las personas tienen de sus habilidades para percibir, asimilar, entender y manejar las emociones propias y las de los demás. Ha sido evaluada con la adaptación al castellano de Fernández-Berrocal, Extremera y Ramos (2004), del Trait MetaMood Scale (TMMS) de Salovey, Mayer, Goldman, Turvey, \& Palfai (1995). Está compuesta por 24 ítems tipo Likert con un rango de puntuación de 1 a 5. Forman tres factores interpersonales, Atención, Claridad y Reparación, con 8 ítems cada uno. El Alfa de Cronbach es 0.86 para el factor Atención, 0.90 para Claridad y 0.86 para Reparación emocional.

\section{Datos sociodemográficos}

Los participantes contestaron a una serie de datos de carácter sociodemográfico como sexo, edad, nivel de estudios (primaria, secundaria, bachillerato o universidad), así como si trabajaban o no y el tipo de trabajo (por cuenta propia, por cuenta ajena o funcionario).

\section{Resultados}

Realizamos el análisis de los datos en función de las hipótesis planteadas.

Hipótesis 1.- Se producirá relación entre Inteligencia Emocional y Cultura del honor.

Para abordar esta hipótesis realizamos varios análisis. En primer lugar, realizamos correlaciones entre todos los factores de Cultura del Honor y componentes de la Inteligencia emocional. En concreto, encontramos que el factor 2, Sociedad y Leyes en torno al honor, correlaciona significativamente con el componente Regulación de las emociones de la inteligencia emocional ( $r=.147 ; \mathrm{p}<.01)$. Esto puede significar que aquellas personas que controlan y regulan más sus emociones otorgan mayor importancia a las normas sociales y, por tanto, son más conscientes de la importancia que se da al honor en su entorno social. Asimismo, personas con mayor regulación emocional no entenderían la cultura del honor como algo individual sino más bien social.

En segundo lugar, realizamos análisis de varianza para comprobar si el nivel de Inteligencia emocional puede predecir la importancia otorgada a la cultura del honor. Para ello, dividimos primero las puntuaciones de Inteligencia emocional en función de la distribución de la muestra y teniendo en cuenta los percentiles 33 y 67 que incluyen a aquellos individuos que puntúan más bajo y los que puntúan más alto de la muestra (véase Extremera, y Fernández-Berrocal, 2005, para un procedimiento similar). Una vez establecida esta división para los tres componentes de la inteligencia emocional, procedimos a realizar un análisis de regresión de cada uno de los factores de cultura del honor introduciendo las tres variables creadas para ver si puntuar alto o bajo en atención, claridad y regulación de las emociones predice algún factor de cultura del honor.

Encontramos que el prestar una alta atención a las emociones predice significativamente la importancia que se le da al honor individual (Factor 1 de cultura del honor) ( $\beta=-0.2 p=0.026$ ). En concreto, los resultados muestran una relación inversa entre ambos factores lo que indica que una alta atención a las emociones provocará una menor importancia dada al honor individual.

Hipótesis 2.- Se producirán diferencias en inteligencia emocional en función de la valoración que los dos miembros de la pareja otorguen a la cultura del honor.

Para dividir la muestra realizamos análisis de conglomerados de K-means para comprobar si las puntuaciones en cultura del honor podían clasificarse mediante agrupaciones en torno a dos puntuaciones (véase López-Zafra, 2008). Obtuvimos dos conglomerados cuyo centro final se situaba en $3.30(n=198)$ y $1.97(n=196)$, por lo que podemos dividir nuestra muestra entre aquellas personas que puntúan alto (grupo 1) y bajo (grupo 2) en la variable cultura del honor. A continuación comprobamos si los dos miembros de cada pareja puntuaron alto o bajo en cultura del honor o bien cada uno tenía una percepción diferente sobre la importancia de la cultura del honor; es decir, un miembro de la pareja puntúa alto en cultura del honor mientras que la otra persona puntúa bajo en 
esta variable. Esta nueva clasificación dio lugar a una nueva variable en la que categorizamos a las personas en función de su pertenencia a una pareja en la que las dos personas tenían una valoración similar sobre cultura del honor (pareja congruente) o distinta sobre la misma (pareja incongruente).

Una vez realizada esta clasificación realizamos diferencias de medias mediante la prueba t-student para comprobar si existían diferencias o no en las dimensiones de inteligencia emocional en función de que los dos miembros de la pareja opinaran lo mismo sobre cultura del honor (congruente) o no (incongruente).

Encontramos, efectivamente, que se producen diferencias significativas en las tres dimensiones de inteligencia emocional en función de tener o no una percepción similar en cultura del honor por parte de los dos miembros de la pareja (véase tabla 1). En todos los casos, cuando ambos miembros de la pareja tenía una percepción similar acerca de la cultura del honor (congruente) puntuaban más alto en cada factor de Inteligencia emocional que cuando cada miembro de la pareja opinaba distinto en torno a la cultura del honor.

Tabla 1.- Dimensiones de Inteligencia emocional y Congruencia en la pareja respecto a la idea de la Cultura del Honor.

\begin{tabular}{|c|c|c|c|}
\hline & Congruente & Incongruente & \multirow{2}{*}{ t-student (prob.) } \\
\cline { 2 - 3 } & $\mathrm{M}(\mathrm{sd})$ & $\mathrm{M}(\mathrm{sd})$ & $2,58(.01)$ \\
\hline $\begin{array}{c}\text { Atención a las } \\
\text { emociones }\end{array}$ & $3,27(.80)$ & $3,04(.81)$ & $2,44(.015)$ \\
\hline Claridad emocional & $3,56(.74)$ & $3,37(.73)$ & $1,98(.049)$ \\
\hline $\begin{array}{c}\text { Regulación de las } \\
\text { emociones }\end{array}$ & $3,46(.78)$ & $3,29(.79)$ & \\
\hline
\end{tabular}

\section{Conclusiones}

En este artículo presentamos un estudio en el que relacionamos cultura del honor e inteligencia emocional, ya que consideramos que ambos conceptos pueden estar relacionados en el proceso de la violencia que se ejerce contra las mujeres en el seno de las relaciones de pareja.

Aunque en la literatura no existen trabajos que relacionen directamente ambos conceptos, sí los hay que vinculan, por un lado, la cultura del honor con la violencia de género (Vandello y Cohen, 2003; López Zafra y Rodríguez Espartal, 2008), con los celos dentro de las relaciones de pareja (Daly y Wilson, 1998; Buss, 2000; Grandon y Cohen, 2002; Puente y Cohen, 2003; López Zafra y Rodríguez Espartal, en prensa; Vandello y Cohen, 2008; Vandello, Cohen, Grandon y Franiuk, en prensa); y, por otro lado, que relacionan la inteligencia emocional con comportamientos violentos hacia la pareja (Winters, Clift y Dutton, 2004) y con el grado de satisfacción en este tipo de relaciones afectivas (Schutte, Malouff, Bobik, Coston, Greeson y Jedlicka, 2001; Smith, Heaven y Ciarrochi, 2008).

Nuestro planteamiento inicial partía de la idea de que un mayor nivel de inteligencia emocional llevaría a otorgar una menor importancia al concepto de cultura del honor. En nuestros resultados comprobamos la existencia de relaciones entre ambos conceptos; en concreto, se produce relación entre "Sociedad y leyes en torno al honor" (factor 2 de cultura del honor) y el componente de "Regulación emocional”. Además, se produce una relación inversa entre la atención emocional y el honor individual, lo que significa que aquellas personas que prestan una atención alta a sus emociones consideran el honor individual como poco importante. Este es un dato positivo de cara a relacionar los conceptos de inteligencia emocional, cultura del honor y violencia de género, pues iría en la línea de que el potenciar la atención emocional de las personas disminuiría la importancia dada 
a la cultura del honor y a los fuertes componentes afectivos que supone, sobre todo para los hombres, el estar inmersos en sociedades asentadas en este tipo de culturas. En esta línea van los trabajos de Shackelford (2005), al hablar de la imagen que deben ofrecer los hombres en las culturas del honor para no dar impresión de debilidad frente al resto del grupo, quizá si se potenciase en estos hombres el prestar atención a sus emociones se conseguiría que esta importancia desmesurada que dan a “su honor”, al honor masculino como lo más importante (López Zafra, 2008), disminuyese en gran medida, lo que repercutiría positivamente en una disminución de la violencia que ejercen contra las mujeres. El hecho de potenciar la atención emocional también resultaría beneficioso para disminuir la importancia otorgada a los roles de género tradicionales, tan importantes en las culturas del honor y decisivos en la consolidación de la violencia de género (López Zafra y Rodríguez Espartal, 2008).

En cuanto a comprobar si se daban diferencias en inteligencia emocional en función de la valoración sobre la cultura del honor que realizaban ambos miembros de una misma pareja, al dividir la muestra en parejas congruentes (valoración similar de la cultura del honor) y parejas incongruentes (diferente valoración de la cultura del honor), encontramos que efectivamente se producían diferencias en las tres dimensiones de inteligencia emocional (atención, claridad y regulación) en función de la igualdad o desigualdad de opinión de los miembros de la pareja respecto al honor. Observamos que las parejas congruentes en la dimensión de cultura del honor puntúan más alto que las incongruentes en las tres dimensiones de la inteligencia emocional consideradas en el estudio; es decir, aquellas parejas en las que los dos miembros otorgan el mismo valor al honor prestan más atención a sus emociones y saben identificarlas y regularlas en mayor medida que las parejas en las que el honor no es valorado en la misma medida por parte de sus miembros. Estos resultados se podrían relacionar con los obtenidos por otros autores que han encontrado que la congruencia entre los miembros de una pareja en cuanto a su inteligencia emocional repercute en la mayor satisfacción en la relación por parte de ambos miembros (Schutte, Malouff, Bobik, Coston, Greeson y Jedlicka, 2001; Smith, Heaven y Ciarrochi, 2008). Si consideramos en conjunto estos resultados, congruencia en cultura del honor e inteligencia emocional y congruencia en inteligencia emocional y mayor satisfacción de pareja, podemos deducir que una menor importancia otorgada a la cultura del honor se asocia con una mayor satisfacción en la relación de pareja (Rodríguez Espartal y López Zafra, 2007) y, además, se podrían considerar en la dirección de los encontrados por diversos autores que comprueban la existencia de una relación inversa entre satisfacción y violencia de género (Stith, Green, Smith y Ward, 2008).

Como se puede observar, potenciar la inteligencia emocional en todas las personas puede resultar muy beneficioso en numerosos aspectos de su vida y de su relaciones con los demás y, en este caso puede repercutir en la satisfacción y bienestar en las relaciones de pareja. Además, tal y como esperábamos, se encuentra una relación entre cultura del honor e inteligencia emocional, lo que puede abrir el camino a una nueva línea de investigación con posibles resultados positivos en el estudio de aspectos relevantes que pudieran explicar la violencia de género, ya que ambos conceptos por separado se han visto relacionados con este tipo de violencia (Winters, Clift y Dutton, 2004; López Zafra y Rodríguez Espartal, 2008). Para ello sería necesario analizar esta relación en parejas en riesgo e incluso incorporar aspectos de entrenamiento en inteligencia emocional para comprobar su impacto en la relación tanto en cuanto a la valoración de la cultura del honor como a la satisfacción con la pareja.

\section{Referencias}

Atchley, J. y Britton, A. (2007). The Hokey Pokey is What It's All About: Words of Wisdom for the Stressed, the Overworked, the Diagnosed, and Those Who Love Them. San Diego: LightenUp Press.

Alcaide, J. (2002). Mal de amores. Muy Interesante (sección Psicología), 253, 44-52.

Amitay, O. y Mongrain, M. (2007). From Emotional Intelligence to Intelligence Choice of Partner.

The Journal of Social Psychology, 147 (4), 325-343. 
Bisquerra, R. (2000). Educación emocional y bienestar. Barcelona: Praxis.

Buss, D. M. (2000). The dangerous passion. New York: Free Press.

Cohen, D. (1996). Law, social policy and violence: the impact of the regional cultures. Journal of Personality and Social Psychology, 70 (5), 961-978.

Cohen, D. y Nisbett, R.E. (1997). Field experiments examining the culture of honor: the role of institutions in perpetuating norms about violence. Personality and Social Psychology Bulletin. vol 23(11) 1188-1199.

Cohen, D.; Nisbett, R.E.; Bowdle, B.F. y Schwarz, N. (1996). Insult, aggression, and the southern culture of honor: an experimental ethnography. Journal of personality and social psychology, 70 (5), 945-960.

Daly, M. y Wilson, M. (1998). Homicide. Hawthorn, New York: Aldine de Gruyter.

Extremera, N., y Fernández-Berrocal, P. (2005). Perceived emocional intelligence and life satisfaction: predictive and incremental validity using the Trait-Meta Mood Scale. Personality and Individual Differences, 39, 937-948.

Extremera, N., Fernández-Berrocal, P. y Salovey, P. (2006). Spanish versión of the Mayer-SaloveyCaruso Emocional Intelligence Test (MSCEIT). Versión 2.0.: Reliabilities, age and gender differences. Psicothema, 18, 42-48.

Fernández-Berrocal, P., Extremera, N. \& Ramos, N. (2004). Validity and reliability of the Spanish modified version of the Trait Meta-Mood Scale. Psychological Reports, 94, 751-755.

Gómez-Jacinto, L., Canto-Ortiz, J. y García-Leiva, P. (2001). Variables moduladoras de las diferencias de sexo en los celos. Revista de Psicología Social, 16 (3), 293-313.

Grandon, R. y Cohen, D. (2002). Violence, jealousy and loyalty in Chile and Canada. Unpublished manuscript, University of Waterloo.

López-Zafra, E. (2007). Elaboración de una escala para medir Cultura del Honor. Revista de Psicología Social, 22 (1), 31-42.

López Zafra, E. (2008). Relación entre Cultura del Honor e Identidad de género: el papel del sexo, edad y nivel de estudios en la predisposición a la violencia. Estudios de Psicología, 29 (2), 209220.

López Zafra, E. y Rodríguez Espartal, N. (2008). Una revisión sobre las consecuencias jurídicas de la cultura del honor en España. Comunicación presentada al IV Congreso de Psicología jurídica y forense. Murcia, 8-10 mayo 2008.

López Zafra, E. y Rodríguez Espartal, N. (en prensa). Relación entre cultura del honor, celos y satisfacción de pareja. Boletín de Psicología.

Mikolajczak, M., Luminet, O., y Menil, C. (2006). Predicting resistance to stress: Incremental validity of trait emotional intelligence over alexithymia and optimism. Psicothema, 18, 79-88.

Puente, S. y Cohen, D. (2003). Jeaolusy and the Meaning (or Nonmeaning of Violence. Personality and social psychology bulletin, 29 (4), 449-460.

Pussent, E. (2006). La felicidad depende más de las relaciones sociales que del dinero o la salud. Obtenido de http://www.gara.net/idatzia/20061028/art186526.php el 17 de abril de 2008.

Rodríguez, N. y López-Zafra, E. (2007). Cultura del honor y relaciones interpersonales en parejas jóvenes universitarias. En C. Guillén y R. Guil (eds). Psicología Social: un encuentro de perspectivas (531-536). Cádiz. Asociación de profesionales de Psicología Social.

Shackelford, T. K. (2005). An Evolutionary Psychological perspective on cultures of honor. Evolutionary Psychology, 3, 381-391. 
Salovey, P. y Grewal, D. (2005).The Science of emotional intelligence. Currents Directions in Psychological Science, 14, 281-285.

Salovey, P., Mayer, J. D., Goldman, S., Turvey, C. \& Palfai, T. (1995). Emotional attention, clarity, and repair: Exploring emotional intelligence using the Trait Meta-Mood Scale. En J. W. Pennebaker (Ed). Emotion, disclosure and health (pp. 125-154). Washington, D. C.: American Psychological Association.

Schutte, N.S., Malouff, J.M., Bobik, C., Coston, T D., Greeson, C., Jedlicka, C. (2001). Emotional Intelligence and Interpersonal relations. Journal of Social Psychology, 141, 523-536.

Smith, L., Heaven, P. y Ciarrochi, J. (2008). Trait emotional intelligence, conflict communication patterns, and relatioship satisfaction. Personality and Individual differences, 44, 1314-1325.

Stith, S.M.; Green, M.N.; Smith D.B. y Ward, D.B. (2008). Marital satisfaction and marital discord as risk markers for intimate partner violence: A meta-analytic review. Journal of Family Violence, 23,3, 149- 161.

Vandello, J. y Cohen, D. (2003). Male Honor and Female Fidelity: Implicit Cultural Scripts That Perpetuate Domestic Violence. Journal of Personality and Social Psychology, 84 (5), 997-1010.

Vandello, J.A. y Cohen, D. (2008). Culture, Gender, and Men’s Intimate Partner Violence. Social and Personality Psychology.

Vandello, J.A.; Cohen, D. y Ransom (2008). U.S. Southern and Northern differences in perceptions of norms about aggression: Mechanisms for the perpetuation of a culture of honor. Journal of cross-cultural psychology, 39, 162-177.

Vandello, J.A.; Cohen, D., Grandon, R., Franiuk, R. (en prensa). Stand by Your Man: Indirect Prescriptions for Honorable Violence and Feminine Loyalty in Canada, Chile, and the United Status. Journal of Cross Cultural Psychology.

Winters, J., Clift, R. y Dutton, D. (2004). An Exploratory Study of Emotional Intelligence and Domestic Abuse. Journal of Family Violence, 19 (5), 255-267.

Yela, C.; Jiménez-Burillo, F. y Sangrador, J.L. (2003). Las dos caras del amor: funciones, mitos, paradojas y renuncias. En S. Worchel, J. Cooper, G. R. Goethals y J.M. Olson (eds.) Psicología Social. Madrid. Thomson. 